\title{
A EDUCAÇÃO FINANCEIRA E AS FINANCAS PESSOAIS SOB A ÓTICA DA BIBLIOMETRIA: UMA ANÁLISE EM EVENTOS DA ADMINISTRAÇÃO NO BRASIL REALIZADOS NO TRIÊNIO 2012- 2014
}

\author{
Natane de Cassia Leivas de Medeiros" \\ Flaviani Souto Bolzan Medeiros**
}

RESUMO: A má gestão dos recursos afeta a saúde financeira dos indivíduos. Por isso, é tão importante, além de conhecer os conceitos da educação financeira, também aplicá-los para organizar as finanças pessoais e familiares. Entretanto, apesar da relevância da discussão dessas temáticas considerando as consequências que a falta desses conhecimentos causa na sociedade como um todo, ainda são poucos os trabalhos nesta área no Brasil. Sendo assim, tendo em vista incentivar novas pesquisas, este artigo teve como objetivo analisar a produção acadêmica acerca da educação financeira e finanças pessoais em eventos da Administração, no Brasil, realizados no triênio 2012-2014 sob a ótica da bibliometria. Para isso, adotou-se uma pesquisa bibliométrica, descritiva e bibliográfica. Os resultados obtidos revelam que a revisão bibliográfica utilizada em sua maioria advém de obras nacionais. Além disso, observou-se que os temas abordados ainda são pouco explorados nos eventos científicos de Administração no país e vêm sendo estudados por um grupo específico de pesquisadores, responsáveis por uma parte considerável das publicações dentro do triênio analisado.

PALAVRAS-CHAVE: Educação Financeira; Finanças Pessoais; Eventos Científicos; Bibliometria.

\section{FINANCIAL EDUCATION AND PERSONAL FINANCES FROM THE POINT OF VIEW OF BIBLIOMETRICS: ANALYSIS IN ADMINISTRATION IN BRAZIL BET WEEN 2012 AND 2014}

\footnotetext{
ABSTRACT: Bad management in resources affects people's financial health. Knowledge on financial education and the manner they should be applied are greatly relevant to organize personal and family finances. In spite of the relevance of the theme and the consequences that the lack of such knowledge causes to society, the-

Graduada em Administração pelo Centro Universitário Franciscano (UNIFRA), Brasil.

E-mail: natanemedeiros@gmail.com

** Doutoranda no Programa de Pós-Graduação em Administração pela Universidade Federal de Santa Maria (UFSM), Brasil.
} 
re are few research works on the subject in Brazil. So that further research would be undertaken, current paper analyzes the academic production on financial education and personal finances in Administration events in Brazil between 2012 and 2014 from the point of view of Bibliometrics. Results of current bibliometric, descriptive and bibliographic research show that bibliographic review focuses on Brazilian production. Themes, only slightly analyzed in scientific events on Administration, are studied by a specific group of researchers who are also the authors of the publications analyzed in the three-year period mentioned above.

KEY WORDS: Financial education; Personal finances; Scientific events; Bibliometrics.

\section{INTRODUÇÃO}

Apesar dos consumidores estarem mais atuantes nas suas relações com as empresas e também estarem mais bem informados (PINTO; CORONEL, 2013), isso não impede que os mesmos enfrentem complicações para honrar seus compromissos financeiros assumidos.

Souza et al. (2013) comentam que aquelas pessoas que não tem um conhecimento mínimo sobre suas finanças pessoais geralmente apresentam como deficiência adesão ao consumismo, gastando acima do que seus rendimentos permitem. E esse consumo exagerado implica em fatores que afeta o cotidiano do indivíduo, um deles é a qualidade de vida, o que resulta no agravo da saúde psicológica desencadeando entre outros problemas a depressão e a insônia, por isso, precisa existir um esforço para o devido controle das finanças pessoais (LUCKE et al., 2014).

Outro ponto que merece destaque envolve as questões de ordem macroeconômica tais como: inflação, taxas de juros e carga tributária que afetam as decisões econômicas da sociedade como um todo, ou seja, elas não alteram apenas os setores empresariais, mas também, o planejamento financeiro das famílias (MEDEIROS; LOPES, 2014).

Sob essa perspectiva, Savoia, Saito e Santana (2007) acrescentam que, na sociedade contemporânea as pessoas precisam ter um domínio de um conjunto amplo de informações de tal forma que isso proporcione uma compreensão lógica e 
sem falhas das forças que interferem no ambiente e nas suas relações com os outros, e isso é adquirido pela educação financeira. Kiyosaki (2011) corrobora que, na Era da Informação, a educação e o aprendizado contínuo são extremamente importantes. Portanto, no mundo de hoje, percebe-se a necessidade da educação financeira ser ofertada tanto nas escolas, para as crianças, como também para os adultos nas instituições de ensino (OPLETALOVÁ, 2015).

Todavia, apesar da importância da educação financeira e das finanças pessoais para a vida dos indivíduos e para a sociedade, decorrente do que o desequilíbrio financeiro pode causar, essas são áreas de estudo ainda muito pouco exploradas no Brasil dentro das Finanças (SOUZA; TORRALVO, 2004; GRÜSSNER, 2007; KRUMMENAUER, 2011; CONCEIÇÃO; BREYER; PRETE, 2013; OLIVEIRA; KASPCZAK; 2013; PUNHAGUI; VIEIRA, 2013; BATISTA; PIGATI; SILVA, 2014).

Diante do exposto, esse artigo tem como problemática responder a seguinte questão: Qual a evolução das publicações sobre educação financeira e finanças pessoais em eventos científicos no país? Para responder à problemática, esse artigo se propõe a analisar a produção acadêmica acerca da educação financeira e finanças pessoais em eventos de Administração, no Brasil, realizados no triênio 2012-2014 sob a ótica da bibliometria.

Considerando a baixa exploração sobre ambos os assuntos no país esse trabalho pretende contribuir com novas discussões a respeito, bem como fazer um levantamento do que vem sendo abordado pelos pesquisadores na área. Para isso, optou-se pelos eventos acadêmicos pelo fato de os mesmos serem comumente utilizados como meio de divulgação de trabalhos gerando aprendizado e novos conhecimentos.

Em termos de estruturação, esse estudo foi organizado em cinco capítulos. Logo após esse de caráter introdutório, o capítulo dois apresenta a revisão bibliográfica contemplando tópicos como educação financeira e também a questão das finanças pessoais e o endividamento. Enquanto que o capítulo três refere-se aos procedimentos metodológicos adotados. Na sequência, o capítulo quatro diz respeito à análise dos resultados. No quinto e último capítulo, por sua vez, constam as considerações finais a partir da pesquisa realizada. 


\section{REVISÃO BIBLIOGRÁFICA}

\subsection{EDUCAÇÃO FINANCEIRA}

Parte-se do entendimento de que todos os meses existem dois acontecimentos em termos financeiros na vida cotidiana: a entrada de dinheiro e o pagamento de contas, deste modo, para se ter um controle, a elaboração de um orçamento é uma prática que se faz necessária (GIORDANI, 2014).

Wohlemberg, Braum e Rojo (2011) afirmam que o orçamento e o planejamento financeiro, desde que bem executados, podem ser ferramentas eficazes no controle tanto das receitas quanto das despesas, contribuindo assim, para uma boa gestão dos recursos ganhos mensalmente.

Sob esse enfoque, Wisniewski (2011) alerta que a ausência do controle no orçamento financeiro, que ocorre muitas vezes pela falta de informação e planejamento financeiro, acaba sendo um dos fatores que prejudica a saúde financeira dos indivíduos em âmbito global.

Por isso, a importância da educação financeira. De acordo com Sousa et al. (2013), a educação financeira é uma maneira de fornecer os conhecimentos e informações sobre comportamentos básicos capazes de contribuir para melhorar a qualidade de vida dos indivíduos e de suas comunidades, isso porque no agregado acaba influenciando toda a economia.

Adicionalmente, Mello (2013) considera a educação financeira uma forma de transferência de conhecimentos e práticas que tem como propósito a conquista de uma melhor qualidade de vida, isso tanto no presente como também no futuro.

Já Potrich, Vieira e Kirch (2014) entendem a educação financeira como um processo que desenvolve habilidades nos indivíduos tornando-os capazes de tomar decisões acertadas, dessa forma, fazendo com que os mesmos tenham uma boa gestão das suas finanças pessoais. Nesse sentido, Kiyosaki (2011) complementa que a chave para um novo mundo é a educação, pois sem uma educação financeira sólida as pessoas não sabem como agir em momentos de crise, tampouco como realizar as mudanças necessárias.

Inclusive, a OECD (2013) lembra que, depois da crise financeira de 2008, surgiu um significativo interesse pela alfabetização financeira por parte de vários 
países ao redor do mundo. Sendo assim, no ano de 2008, foi criado pela OECD a International Network on Financial Education (INFE) - Rede Internacional de Educação Financeira - iniciativa apoiada pelos líderes do G20 (G20, 2012).

Conforme dados divulgados pela OECD (2015), atualmente, mais de 240 instituições públicas de mais de 110 países aderiram ao INFE, um órgão que visa promover e facilitar a cooperação internacional entre os decisores políticos e outras partes interessadas sobre questões de educação financeira em nível mundial.

Segundo Grifoni e Messy (2012), os governantes estão cada vez mais preocupados com os baixos níveis de educação financeira dos seus cidadãos, pois reconhecem que boas habilidades financeiras seriam capazes de capacitar as pessoas para tomarem as melhores decisões, em um mercado cada vez mais complexo. $\mathrm{O}$ fato é que independente do valor auferido como salário todos devem entender os conceitos básicos de educação financeira, pois é importante encontrar uma forma de ter uma vida financeira equilibrada, ou seja, equacionar as despesas ao rendimento e, ainda, ter uma sobra para investir ou poupar (GUIMARÃES, 2013).

\subsection{FINANÇAS PESSOAIS E O ENDIVIDAMENTO}

As finanças pessoais, na visão de Fernandes, Monteiro e Santos (2012), tem como objetivo maximizar a riqueza do indivíduo, passando por decisões que envolvem desde financiamento, investimento, consumo até avaliação de risco e de retorno que estejam em conformidade com os objetivos pessoais.

Em outras palavras, as finanças pessoais preocupam-se com o planejamento da renda pessoal determinando quanto deve ser destinado às despesas, qual a parcela que deve ir para a poupança, para alguma eventualidade e situação de crise e, ainda, quais os investimentos que podem vir a ser concretizados visando obter um retorno, e assim, realizar os sonhos materiais (LUCENA; MARINHO, 2013).

Marques, Souza e Pessoa (2014) acrescentam que as finanças pessoais tratam ainda dos problemas envolvendo o orçamento familiar, bem como as maneiras de fazer uso dos créditos disponíveis no mercado financeiro, as aplicações vantajosas, e também, como diversificar as fontes de renda pessoal. Cabe ressaltar que a falta desses conceitos básicos sobre finanças pessoais conduz os indivíduos, de 
modo fácil, ao endividamento, o que por sua vez, resulta na perda da qualidade de vida (SOUZA et al., 2013).

Na opinião de Grüssner (2007), a facilidade de acesso ao crédito e o descontrole financeiro são alguns dos fatores que conduzem as pessoas ao endividamento. Avdzejus, Santos e Santanta (2012) reforçam que o endividamento dos indivíduos é decorrente desse descontrole financeiro, por isso, recomendam que antes de assumir um compromisso de compra, que se avalie a real necessidade de adquirir aquele bem.

Mas, além dessa facilidade de acesso ao crédito, Figueira (2013) atribui essa questão do endividamento também em função do aumento do otimismo da população. De acordo com os dados divulgados pelo Instituto de Pesquisa Econômica Aplicada (IPEA, 2012), o Índice de Expectativas das Famílias (IEF) que revela o otimismo das famílias a respeito da situação socioeconômica do país, apontou 68,5\% no corrente ano.

Ademais, outro ponto que vem sendo discutido é o sobre-endividamento das famílias, caso em que o indivíduo não se sente possibilitado para quitar seus compromissos financeiros assumidos (FLORES; CAMPARA; VIEIRA, 2012). Além disso, vale destacar que os jovens em particular, estão tendo que fazer suas escolhas financeiras cada vez mais cedo (LUSARDI; MITCHELL; CURTO, 2010).

Em vista disso, percebe-se o quanto as finanças pessoais são importantes para determinar o sucesso financeiro de uma pessoa, auxiliando-a na gestão dos seus recursos para que a mesma possa ter satisfação tanto pessoal quanto econômica (KAPOOR; DALABAY; HUGHES, 2012; ISMAIL et al., 2013).

Contribuindo nesse sentido, Mette e Matos (2014) apontam que o incentivo aos programas de alfabetização financeira ou educação financeira tem sido considerado também um meio de auxiliar a população para um consumo consciente.

Marcolin e Abraham (2006) reforçam o exposto, ao levar em conta que está cada vez maior a acessibilidade a produtos e serviços financeiros, deste modo, ter certo entendimento sobre educação financeira se faz necessário. Portanto, para Gomes e Sorato (2010), tão importante quanto ter uma atividade que proporcione uma estabilidade financeira é adquirir o hábito e a cultura de organizá-la. 


\section{METODOLOGIA}

Este artigo foi desenvolvido a partir de uma pesquisa bibliográfica quanto aos meios, do tipo descritiva quanto aos fins, adotando a técnica da bibliometria. Deste modo, a respeito da pesquisa bibliográfica, esta se refere ao levantamento teórico sobre o tema abordado com o objetivo de levantar informações sobre o assunto (MICHEL, 2009).

Acerca da pesquisa descritiva, Appolinário (2011) explica que este limita-se a descrever o fenômeno analisado, sem interferir nas relações de causalidade entre as variáveis consideradas. Já a pesquisa bibliométrica tem como propósito acompanhar, mapear e avaliar as produções científicas, principalmente em relação às tendências temáticas e metodológicas dos trabalhos (ESPEJO et al., 2009).

Os dados foram coletados a partir de uma busca realizada nos anais dos eventos ADMpg, CNEG, Convibra, Enangrad, SEGeT e SemeAd. Para a seleção dos trabalhos filtrou-se aqueles publicados no triênio 2012-2014 que continham as palavras finanças pessoais, educação financeira, alfabetização financeira, planejamento financeiro, planejamento e controle financeiro e/ou saúde financeira no título ou palavras-chaves. Ao todo foram encontrados 33 artigos.

Logo após esta etapa, as informações referentes aos artigos foram tabuladas com auxílio do Microsoft Excel ${ }^{\circledR}$. Destaca-se que a fase de coleta e análise dos dados ocorreu entre os meses de abril a julho de 2015. Em relação à escolha dos eventos, isso se deve ao fato de que os temas abordados ainda são pouco explorados no meio acadêmico, deste modo, com o uso de diversos eventos foi possível enriquecer a amostra aqui considerada para fins de análise. Além disso, optou-se por eventos de renome nacional, uma vez que são conhecidos por sua tradição e qualidade das publicações.

\section{ANÁLISE DOS RESULTADOS}

Nesta seção apresentam-se os resultados obtidos através da análise realizada nos anais dos eventos ADMpg, CNEG, Convibra, Enangrad, SEGe'T e SemeAd. Com o 
intuito de melhor apresentá-los, esta seção encontra-se subdividida em dois tópicos, a saber: informações sobre os artigos publicados no triênio 2012 a 2014 e informações sobre os autores e coautores.

\subsection{INFORMAÇÕES SOBRE OS ARTIGOS PUBLICADOS NO TRIÊNIO 2012-2014}

A partir da busca realizada nos anais de cada evento, os dados obtidos estão distribuídos no Gráfico 1, de acordo com o ano de publicação.

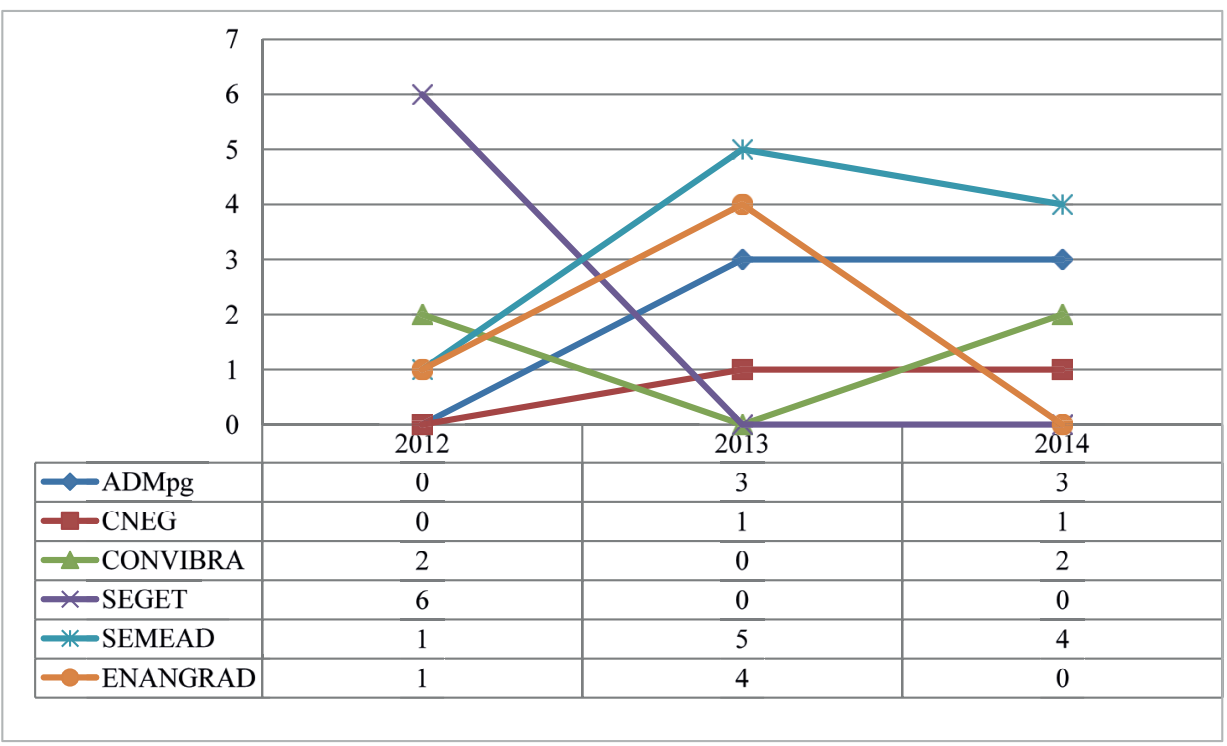

Gráfico 1. Número de artigos publicados em cada evento no triênio 2012 a 2014 Fonte: Elaborado pelos autores com base nos dados da pesquisa (2015).

Observa-se no Gráfico 1 que, por um lado, em alguns anos os eventos não tiveram trabalhos publicados sobre as temáticas finanças pessoais e educação financeira em seus anais. Por outro lado, o SemeAd aparece com um total de 10 artigos, obtendo o maior número de publicações no triênio, em contrapartida, o CNEG apresentou o menor número, com apenas 02 trabalhos. Analisando o triênio, verifica-se que 2012 e 2014 apresentam a mesma quantidade de trabalhos, ou seja, 10 artigos e o ano de 2013 destaca-se com 13 publicações. O evento SEGeT, por sua vez, apresentou 6 artigos em sua totalidade, no entanto, todos foram publicados nos 
anais de 2012, nos anos seguintes não houve publicação de novos trabalhos acerca dos temas aqui considerados.

Retornando ao SemeAd, observa-se que este é o único evento em que houve publicação em todos os anos analisados. Isso evidencia que os temas finanças pessoais e educação financeira ainda são pouco explorados nos eventos de Administração no Brasil. A seguir, o Quadro 1 foi elaborado com base nos principais autores utilizados como referência nos trabalhos de finanças pessoais e educação financeira. Optou-se por classificá-los por evento, em ordem decrescente de quantidade de citações. Para tanto, criou-se a coluna nacionalidade que tem por objetivo apresentar a origem dos principais autores.

Quadro 1. Autores mais referenciados em cada evento

(Continua)

\begin{tabular}{|c|l|c|c|c|}
\hline \multirow{5}{*}{ Evento } & \multicolumn{1}{|c|}{ Autor } & Nacionalidade & Citação & Percentual \\
\hline \multirow{5}{*}{ ADMpg } & CERBASI, Gustavo P. & Brasil & 4 & $2,45 \%$ \\
\cline { 2 - 5 } & FRANKENBERG, Louis & Brasil & 4 & $2,45 \%$ \\
\cline { 2 - 5 } & GITMAN, Lawrence J. & Estados Unidos & 3 & $1,84 \%$ \\
\cline { 2 - 5 } & IUDÍCIBUS, Sérgio de & Brasil & 3 & $1,84 \%$ \\
\hline \multirow{5}{*}{ CNEG } & CERBASI, Gustavo P. & Brasil & 4 & $5,33 \%$ \\
\cline { 2 - 5 } & TOLOTTI, Márcia & Brasil & 2 & $2,67 \%$ \\
\cline { 2 - 5 } & MACEDO JUNIOR, Jurandir Sell & Brasil & 2 & $2,67 \%$ \\
\hline \multirow{5}{*}{ CONVIBRA } & CERBASI, Gustavo P. & Brasil & 5 & $4,71 \%$ \\
\cline { 2 - 5 } & ZERRENNER, Sabrina Arruda & Brasil & 4 & $3,77 \%$ \\
\cline { 2 - 5 } & KUMAR, Vinay & Índia & 3 & $2,83 \%$ \\
\cline { 2 - 5 } & DAY, George S. & Estados Unidos & 3 & $2,83 \%$ \\
\hline \multirow{5}{*}{ ENANGRAD } & LUSARDI, Annamaria & Itália & 5 & $2,49 \%$ \\
\cline { 2 - 5 } & SAITO, André Taue & Brasil & 4 & $1,99 \%$ \\
\cline { 2 - 5 } & MATTA, Rodrigo Octávio Beton & 3 & $1,49 \%$ \\
\cline { 2 - 5 } & CHEN, Haiyang & Estados Unidos & 3 & $1,49 \%$ \\
\cline { 2 - 5 } & SANTANA, Flávia de Angelis & Brasil & 3 & $1,49 \%$ \\
\cline { 2 - 5 } & SAVOIA, José Roberto Ferreira & Brasil & 3 & $1,49 \%$ \\
\cline { 2 - 5 } & VOLPE, Ronald P. & Estados Unidos & 3 & $1,49 \%$ \\
\hline
\end{tabular}


(Conclusão)

\begin{tabular}{|c|l|c|c|c|}
\hline Evento & \multicolumn{1}{|c|}{ Autor } & Nacionalidade & Citação & Percentual \\
\hline \multirow{5}{*}{ SEGET } & CERBASI, Gustavo P. & Brasil & 5 & $4,24 \%$ \\
\cline { 2 - 5 } & FRANKENBERG, Louis & Brasil & 5 & $4,24 \%$ \\
\cline { 2 - 5 } & CHEROBIM, Ana Paula Mussi Szabo & Brasil & 4 & $3,39 \%$ \\
\cline { 2 - 5 } & HALFELD, Mauro & Brasil & 4 & $3,39 \%$ \\
\cline { 2 - 5 } & PERETTI, Luiz Carlos & Brasil & 4 & $3,39 \%$ \\
\hline \multirow{5}{*}{ SEMEAD } & LUSARDI, Annamaria & Itália & 11 & $2,15 \%$ \\
\cline { 2 - 6 } & MITCHELL, Olivia. S. & Estados Unidos & 7 & $1,37 \%$ \\
\cline { 2 - 6 } & SAVOIA, José Roberto Ferreira & Brasil & 6 & $1,17 \%$ \\
\cline { 2 - 6 } & ZERRENNER, Sabrina Arruda & Brasil & 6 & $1,17 \%$ \\
\hline
\end{tabular}

Fonte: Elaborado pelos autores com base nos dados da pesquisa (2015).

Deste modo, observa-se no Quadro 1 que a maioria, ou seja, 70\% dos principais autores referenciados são nacionais. Se a análise considerar as citações como um todo, independentemente do evento, percebe-se que Gustavo P. Cerbasi é o autor mais referenciado com 25 citações, seguido por Annamaria Lusardi, com 18 citações. Salienta-se que a coluna percentual é calculada com base no evento a qual o artigo pertence, com destaque novamente para Cerbasi.

Além do levantamento sobre os principais autores verificou-se também as principais obras utilizadas como revisão bibliográfica para o embasamento dos artigos publicados no triênio. Tais informações são expostas no Quadro 2.

Quadro 2. Principais obras referenciadas em cada evento

(Continua)

\begin{tabular}{|c|l|c|c|c|c|}
\hline Evento & \multicolumn{1}{|c|}{ Obra } & Tipo & Ano & Autor & Citações \\
\hline \multirow{2}{*}{ ADMpg } & $\begin{array}{l}\text { Seu futuro financeiro, você é } \\
\text { o maior responsável: como } \\
\text { planejar suas finanças pes- } \\
\text { soais para toda a vida }\end{array}$ & Livro & 1999 & FRANKENBERG, L. & 3 \\
\cline { 2 - 6 } & $\begin{array}{l}\text { Casais inteligentes enrique- } \\
\text { cem juntos }\end{array}$ & Livro & 2004 & CERBASI, G. P. & 3 \\
\hline
\end{tabular}


(Conclusão)

\begin{tabular}{|c|c|c|c|c|c|}
\hline \multirow[t]{2}{*}{ CNEG } & $\begin{array}{l}\text { A árvore do dinheiro } \\
\text { guia para cultivar a sua } \\
\text { independência financeira }\end{array}$ & Livro & 2007 & MACEDO JR, J. S. & 2 \\
\hline & $\begin{array}{l}\text { As armadilhas do consumo: } \\
\text { acabe com o endividamento }\end{array}$ & Livro & 2007 & TOLOTTI, Márcia. & 2 \\
\hline \multirow{2}{*}{ CONVIBRA } & Pesquisa de marketing & Livro & 2007 & $\begin{array}{l}\text { AAKER, A. D.; KUMAR, } \\
\text { V.; DAY, G. S. }\end{array}$ & 6 \\
\hline & $\begin{array}{l}\text { Casais inteligentes enrique- } \\
\text { cem juntos }\end{array}$ & Livro & 2004 & CERBASI, Gustavo P. & 3 \\
\hline ENANGRAD & $\begin{array}{l}\text { Paradigmas da educação fi- } \\
\text { nanceira no Brasil }\end{array}$ & $\begin{array}{l}\text { Periódi- } \\
\quad \text { co }\end{array}$ & 2007 & $\begin{array}{l}\text { SAVOIA, J. R. F.; SAI- } \\
\text { TO, A. T.; SANTANA, } \\
\text { F. de A. }\end{array}$ & 3 \\
\hline \multirow{3}{*}{ SEGET } & $\begin{array}{l}\text { Investimentos: como admi- } \\
\text { nistrar melhor o seu dinhei- } \\
\text { ro }\end{array}$ & Livro & 2001 & HALFELD, M. & 4 \\
\hline & $\begin{array}{l}\text { Finanças pessoais: conhecer } \\
\text { para enriquecer! }\end{array}$ & Livro & 2010 & $\begin{array}{l}\text { CHEROBIM, A. P. M. } \\
\text { S.; ESPEJO, M. M. dos } \\
\text { S. B. }\end{array}$ & 3 \\
\hline & $\begin{array}{l}\text { Seu futuro financeiro: você é } \\
\text { o maior responsável }\end{array}$ & Livro & 1999 & FRANKENBERG, L. & 3 \\
\hline \multirow{4}{*}{ SEMEAD } & $\begin{array}{l}\text { A influência da educação } \\
\text { financeira nas decisões de } \\
\text { consumo e investimento dos } \\
\text { indivíduos }\end{array}$ & $\begin{array}{l}\text { Anais de } \\
\text { eventos }\end{array}$ & 2011 & $\begin{array}{l}\text { LUCCI, C. R.; ZER- } \\
\text { RENNER, S.; VERRO- } \\
\text { NE, M. A. G.; SANTOS, } \\
\text { S. C. dos. }\end{array}$ & 4 \\
\hline & $\begin{array}{l}\text { Educação financeira e de- } \\
\text { cisões de consumo, inves- } \\
\text { timento e poupança: uma } \\
\text { análise dos alunos de uma } \\
\text { universidade pública do nor- } \\
\text { te do Paraná }\end{array}$ & $\begin{array}{l}\text { Periódi- } \\
\quad \text { co }\end{array}$ & 2011 & $\begin{array}{l}\text { VIEIRA, S. F. A.; BA- } \\
\text { TAGLIA, R. T. M.; SE- } \\
\text { REIA, V. J. }\end{array}$ & 4 \\
\hline & $\begin{array}{l}\text { Educação financeira para um } \\
\text { Brasil sustentável evidências } \\
\text { da necessidade de atuação } \\
\text { do Banco Central do Brasil } \\
\text { em educação financeira para } \\
\text { o cumprimento de sua mis- } \\
\text { são }\end{array}$ & $\begin{array}{l}\text { Periódi- } \\
\quad \text { co }\end{array}$ & 2012 & $\begin{array}{l}\text { ARAÚJO, F. de A. L.; } \\
\text { SOUZA, M. A. P. de. }\end{array}$ & 4 \\
\hline & $\begin{array}{l}\text { Finanças pessoais: um estu- } \\
\text { do de caso com servidores } \\
\text { públicos }\end{array}$ & $\begin{array}{l}\text { Anais de } \\
\text { eventos }\end{array}$ & 2009 & $\begin{array}{l}\text { CLAUDINO, L. P.; NU- } \\
\text { NES, M. B.; SILVA, F. } \\
\text { C. da. }\end{array}$ & 4 \\
\hline
\end{tabular}

Fonte: Elaborado pelos autores com base nos dados da pesquisa (2015). 
Cabe frisar que para a elaboração do referido Quadro 2 levou-se em conta tanto a obra como o seu respectivo autor, o seu tipo, o ano, bem como o evento ao qual se refere. Na coluna citações, encontra-se o número de vezes em que a obra foi utilizada no referido evento. Sendo assim, é possível analisar que independente do evento as principais obras referenciadas tratam-se de livros. A única exceção fica a cargo do SemeAd, uma vez que as obras mais utilizadas por seus autores não incluem livros, mas sim, periódicos e anais de eventos.

Em seguida, ao analisar todas as palavras-chave abordadas nos 33 trabalhos salienta-se que diversas obtiveram destaque como consumo, orçamento doméstico e crédito. Porém, as palavras endividamento, educação financeira e finanças pessoais foram as mais adotadas nos artigos aqui considerados para fins de análise. No tópico a seguir serão abordadas informações referentes aos autores e coautores dos artigos analisados.

\subsection{INFORMAÇÕES SOBRE OS AUTORES E COAUTORES}

Com relação às informações sobre os autores na quantificação acerca do gênero o masculino foi a maioria, representando 54\% do total. Em termos de evento a distribuição permaneceu nessa ordem: SEMEAD (16); SEGET (11); ENANGRAD (10); ADMpg (9); CNEG (3); e CONVIBRA (2). Enquanto no gênero feminino a distribuição ficou da seguinte forma: SEMEAD (13); ADMpg (8); ENANGRAD e SEGeT ambos com 7 em cada; CONVIBRA (5); e CNEG (3).

Na sequência, o Gráfico 2 apresenta a média de autores por artigo conforme o evento e o ano. Verifica-se que em alguns anos, a média encontrada é zero, visto que neste ano não houve publicações sobre o tema em estudo no referido evento. 


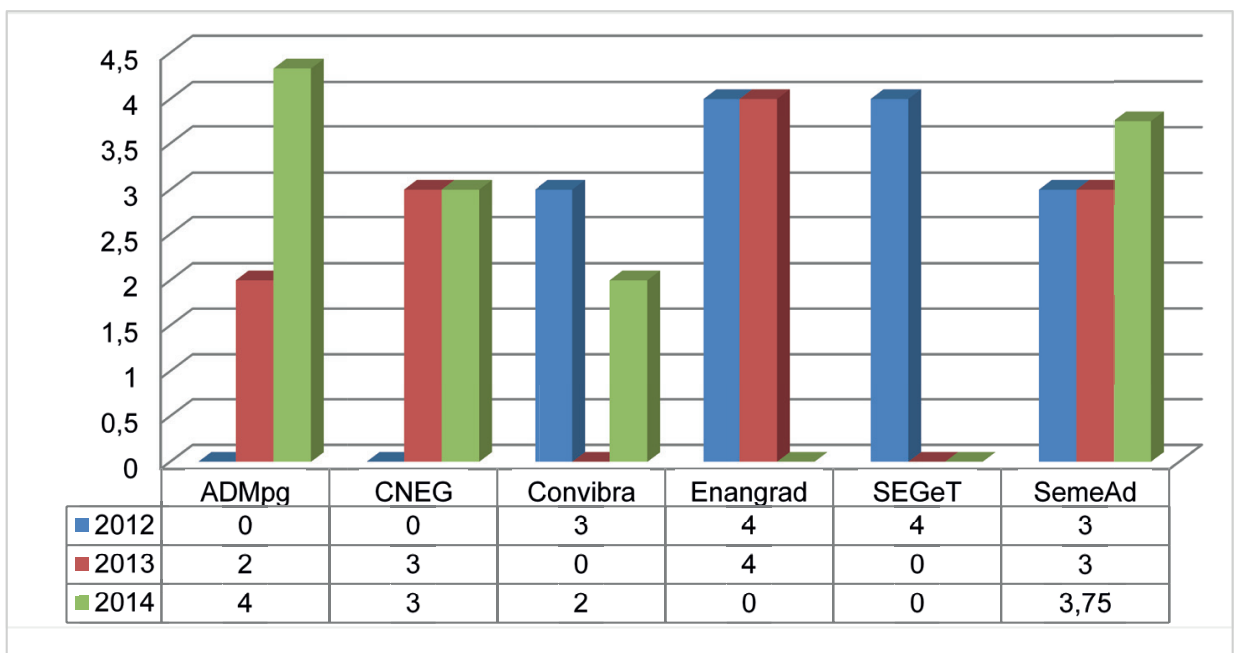

Gráfico 2. Média de autores por artigo de acordo com o ano e evento Fonte: Elaborado pelos autores com base nos dados da pesquisa (2015).

Conforme visualiza-se no Gráfico 2, a menor média encontrada é de 02 autores, logo, evidencia-se que não houve trabalhos publicados com apenas 01 autor, ou seja, todos os artigos possuíam no mínimo 01 autor e 01 coautor. No ano de 2014, o evento ADMpg apresenta a maior média, obtendo 4,33 autores por trabalho, próximo do limite máximo de autores permitidos por artigo, no caso 05 autores.

O Quadro 3, por sua vez, remete às Instituições de Ensino Superior (IES) na qual os autores dos trabalhos encontravam-se vinculados. Salienta-se que a classificação foi realizada por evento e, em seguida, por ordem decrescente de número de autores, desta forma, algumas IES se repetem. A coluna "autores vinculados" expõe a quantidade de autores e coautores que possuem vínculo com a referida instituição.

Para tanto, vale destacar que a Universidade Federal de Brasília possui o maior número de autores vinculados, desconsiderando o evento de origem, uma vez que está vinculada a 15 autores somando os eventos SemeAd e Enangrad, em que aparece em primeira posição entre as IES. 
Quadro 3. Instituição de vínculo dos autores

(Continua)

\begin{tabular}{|c|c|c|c|}
\hline Evento & IES & UF & $\begin{array}{c}\text { Autores } \\
\text { vinculados }\end{array}$ \\
\hline \multirow{7}{*}{ ADMpg } & Universidade Comunitária Regional de Chapecó & $\mathrm{SC}$ & 8 \\
\hline & Serviço Nacional de Aprendizagem Comercial & $\mathrm{SC}$ & 3 \\
\hline & Universidade Federal de Santa Maria & RS & 2 \\
\hline & Faculdade União & PR & 2 \\
\hline & Fundação Escola do Comércio Álvares Penteado & SP & 1 \\
\hline & Fundação Hermínio Ometto & SP & 1 \\
\hline & Universidade Federal da Fronteira Sul & SC & 1 \\
\hline \multirow{2}{*}{ CNEG } & Universidade Federal de Mato Grosso & MT & 3 \\
\hline & Faculdade do Espírito Santo & ES & 3 \\
\hline \multirow{3}{*}{ CONVIBRA } & Universidade Federal de Uberlândia & MG & 3 \\
\hline & Faculdade Católica do Tocantins & TO & 2 \\
\hline & Centro Universitário do Leste de Minas & MG & 2 \\
\hline \multirow{6}{*}{ ENANGRAD } & Universidade Católica de Brasília & DF & 7 \\
\hline & Universidade Federal de Santa Maria & RS & 4 \\
\hline & Universidade Federal de Juiz de Fora & MG & 2 \\
\hline & Universidade Corporativa do Banco Central do Brasil & $\mathrm{DF}$ & 1 \\
\hline & Faculdade Campo Limpo Paulista & SP & 1 \\
\hline & Faculdade Alves Farias & GO & 1 \\
\hline \multirow{7}{*}{ SEGET } & Universidade Nove de Julho & SP & 6 \\
\hline & Universidade do Estado de Santa Catarina & SC & 4 \\
\hline & Universidade do Vale do Itajaí & SC & 3 \\
\hline & Universidade Paulista & SP & 2 \\
\hline & FATEC - Universidade Livre de São Paulo & SP & 1 \\
\hline & Fundação Getúlio Vargas & SP & 1 \\
\hline & Faculdade do Litoral Sul Paulista & SP & 1 \\
\hline
\end{tabular}


(Conclusão)

\begin{tabular}{|l|l|c|c|}
\hline \multirow{5}{*}{} & Universidade Católica de Brasília & DF & 8 \\
\cline { 2 - 4 } & Universidade Federal de Santa Maria & RS & 5 \\
\cline { 2 - 4 } & Universidade Federal de Uberlândia & MG & 5 \\
\cline { 2 - 4 } & Universidade Presbiteriana Mackenzie & SP & 3 \\
\cline { 2 - 4 } & Universidade Regional do Estado do Rio Grande do Sul & RS & 2 \\
\cline { 2 - 4 } & Universidade Estadual de Londrina & PR & 2 \\
\cline { 2 - 4 } & Faculdade do Vale do Ipojuca & PE & 1 \\
\cline { 2 - 5 } & Universidade Federal da Paraíba & PA & 1 \\
\cline { 2 - 5 } & Instituto Federal do Triângulo Mineiro & MG & 1 \\
\cline { 2 - 5 } & Universidade Regional de Blumenau & SC & 1 \\
\cline { 2 - 5 } & Universidade Regional do Noroeste do Estado do Rio Gran- & RS & 1 \\
\hline & de do Sul & & \\
\cline { 2 - 5 } & Universidade Corporativa do Banco Central do Brasil & DF & 1 \\
\hline \multicolumn{1}{|c}{ Total } & & 96 \\
\hline
\end{tabular}

Fonte: Elaborado pelos autores com base nos dados da pesquisa (2015).

Em seguida, verifica-se no Quadro 3 que a Universidade Federal de Santa Maria está vinculada a 11 autores no total, estando presente nos eventos ADMpg, Enangrad e SemeAd. Considerando-se os Estados onde as instituições estão localizadas, ressalta-se que São Paulo (9) seguido por Santa Catarina (7) são as unidades federativas que apresentam o maior número de IES com autores vinculados no triênio. Os autores e coautores que mais publicaram sobre o tema finanças pessoais e educação financeira, nos anais dos eventos analisados, encontram-se dispostos no Quadro 4. Este, por sua vez, foi composto por todos os autores e coautores que possuem dois ou mais trabalhos. 
Quadro 4. Autores com duas ou mais publicações por evento

\begin{tabular}{|l|l|c|c|}
\hline \multicolumn{1}{|c|}{ Evento } & \multicolumn{1}{|c|}{ Autor } & Artigos & $\begin{array}{c}\text { Percen- } \\
\text { tual }\end{array}$ \\
\hline ENANGRAD / SEMEAD & Alberto Shigueru Matsumoto & 5 & $4,46 \%$ \\
\hline ENANGRAD / SEMEAD & Abdelkader Bourahli & 3 & $2,68 \%$ \\
\hline ENANGRAD / SEMEAD & Kelmara Mendes Vieira & 3 & $2,68 \%$ \\
\hline ENANGRAD / SEMEAD & Ana Luiza Paraboni & 2 & $1,78 \%$ \\
\hline ENANGRAD / SEMEAD & Idalberto José das Neves Júnior & 2 & $1,78 \%$ \\
\hline ENANGRAD / SEMEAD & Jéssica Pulino Campara & 2 & $1,78 \%$ \\
\hline ENANGRAD / SEMEAD & Rodrigo Octavio Beton Matta & 2 & $1,78 \%$ \\
\hline SEGET & Armindo Aparecido Evangelista & 2 & $1,78 \%$ \\
\hline SEGET & Dinorá Baldo de Faveri & 2 & $1,78 \%$ \\
\hline CONVIBRA & Josilene da Silva Barbosa & 2 & $1,78 \%$ \\
\hline SEGET & Marilei Kroetz & 2 & $1,78 \%$ \\
\hline CONVIBRA & Marli Auxiliadora da Silva & 2 & $1,78 \%$ \\
\hline SEGET & Nelson Alonso Junior & 2 & $1,78 \%$ \\
\hline SEMEAD & Pablo Rogers & 2 & $1,78 \%$ \\
\hline ADM PG & Paulo Sérgio Jordani & 2 & $1,78 \%$ \\
\hline SEMEAD & Poliana Cristina de Oliveira Cristo Diniz & 2 & $1,78 \%$ \\
\hline CONVIBRA & Rejane Alexandrina Domingues Pereira do & 2 & $1,78 \%$ \\
\hline ADM PG & Prado & $1,78 \%$ \\
\hline SEGET & Vodrigo Barichello & $1,78 \%$ \\
\hline SEGET & Vera Lucia Chaves Alonso & $1,78 \%$ \\
\hline & & 2 & \\
\hline
\end{tabular}

Fonte: Elaborado pelos autores com base nos dados da pesquisa (2015).

Cabe destacar que o Quadro 4 considerou os principais autores, analisando os dados como um todo, e não por evento, como ocorreu em outras análises decorridas nesse estudo. Sendo assim, inicialmente, é possível constatar que os sete 
principais autores tiveram seus estudos publicados nos mesmos eventos: Enangrad e SemeAd. Os demais autores destacaram-se por sua produção em apenas 01 evento.

Deste modo, tem-se Alberto Shigueru Matsumoto como principal autor acerca dos temas abordados, representando 4,46\% do total de autores e coautores. Na sequência, os pesquisadores Abdelkader Bourahli e Kelmara Mendes Vieira possuem 03 trabalhos cada, representando 2,68\% cada. Ao levar-se em conta que o total de autores e coautores (independente da repetição) somou 112 e o total de 45 apresentado no Quadro 5 indica que aproximadamente $40 \%$ dos autores publicaram mais de um artigo, pode-se dizer que os temas aqui analisados encontram-se sendo trabalhados por um grupo restrito de autores no país.

$\mathrm{Na}$ Figura 1 segue a rede de relacionamentos a fim de mostrar a interação existente entre os autores.

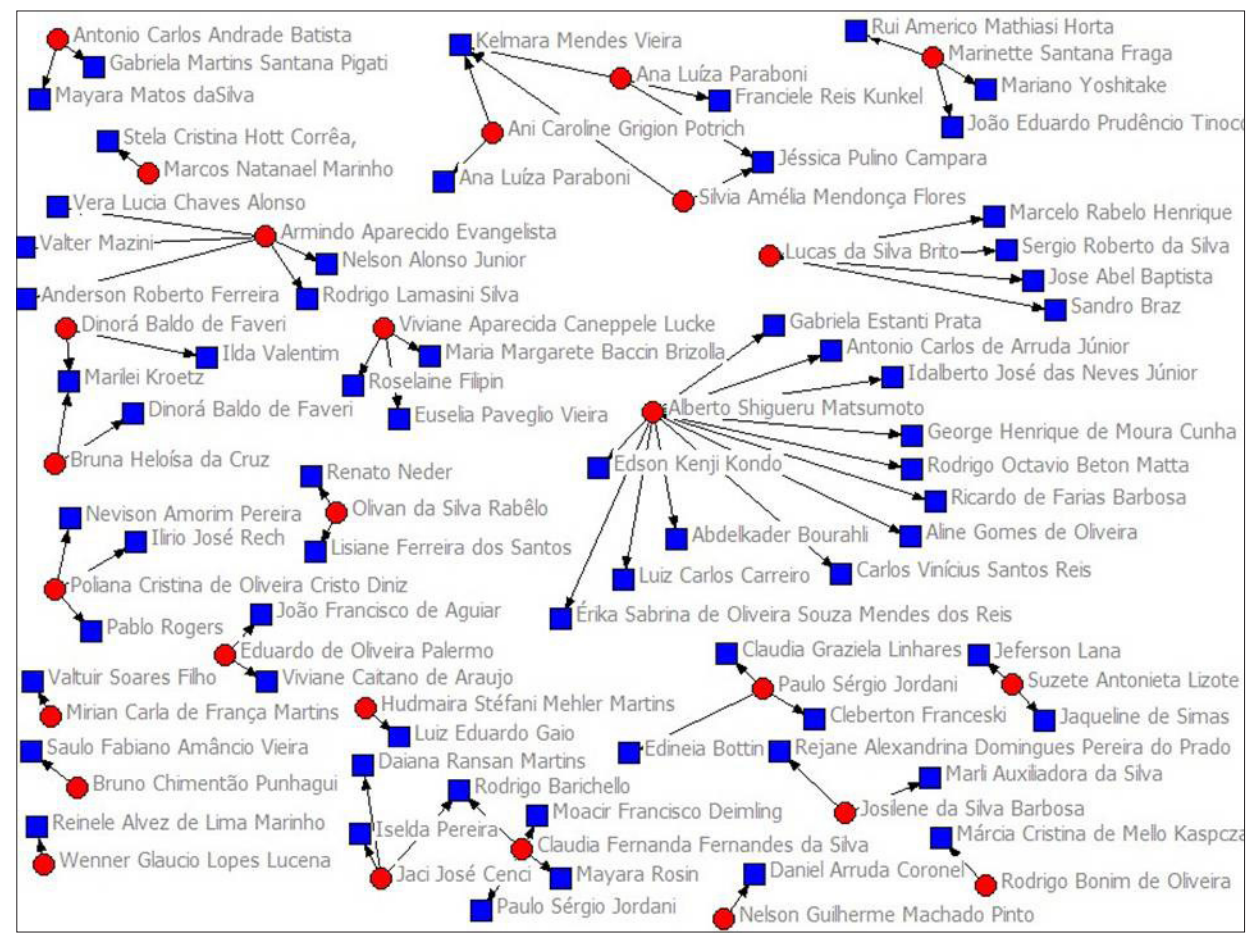

Legenda: $\bigcirc$ Autor $\square$ Coautor

Figura 1. Rede de autores

Fonte: Elaborado pelos autores com base nos dados da pesquisa (2015). 
Conforme já apresentado no decorrer deste estudo, alguns autores possuem trabalhos publicados em eventos distintos, no entanto, na Figura 1 não foi considerado tais eventos para a confecção da rede de relacionamentos. Portanto, esta visa analisar a interação existente entre os autores, independente do evento originário.

Analisando as três principais redes apresentadas na Figura 1, nota-se que a mesma inicia-se pela rede do autor Alberto Shigueru Matsumoto, onde este possui 05 artigos publicados estando vinculado a 12 coautores, porém, esta rede não possui interações visto que ele é o autor em todos os trabalhos. Em seguida, tem-se a rede formada pelas autoras Ana Luíza Paraboni, Sílvia Amélia Mendonça Flores e Ani Caroline Grigion Potrich, onde cada uma encontra-se como autora principal. A maior interação desta rede é a coautora Kelmara Mendes Vieira, vinculada ao trabalho das três autoras, seguida pela coautora Jéssica Pulino Campara, vinculada a dois trabalhos desta rede, além disso, a autora Ana Luíza Paraboni encontra-se vinculada como coautora no artigo de Ani Caroline Grigion Potrich.

E por fim, a rede das autoras Bruna Heloísa da Cruz e Dinorá Baldo de Faveri apresentam como elo a coautora Marilei Kroetz, além disso, a autora Dinorá está vinculada como coautora no trabalho de Bruna Heloísa da Cruz. Os demais casos não apresentaram redes com interações, apenas o vínculo simples dos autores no referido trabalho.

\section{CONSIDERAÇÕES FINAIS}

Considerando a importância da alfabetização financeira, este trabalho teve como objetivo analisar a produção acadêmica acerca da educação financeira e finanças pessoais em eventos de Administração, no Brasil, realizados no triênio 2012-2014 sob a ótica da bibliometria. Para este estudo, foram analisados 33 artigos publicados nos anais dos eventos ADMpg, CNEG, Convibra, Enangrad, SEGeT e SemeAd, sendo este último o evento que possui a maior produção acadêmica sobre os temas abordados.

A análise foi dividida em dois blocos, sendo o primeiro focado em informações relacionadas aos artigos, e o segundo, visando abordar as informações 
sobre os autores. Durante a análise, verificou-se que o tema é bastante trabalhado por autores nacionais, inclusive os mais referenciados são brasileiros. Considerando as obras utilizadas de um modo geral, observou-se que os principais autores citados são Gustavo P. Cerbasi, seguido por Annamaria Lusardi. Nesse sentido, as obras preferidas pelos autores são os livros, exceto o evento SemeAd que utilizou apenas periódicos e anais em eventos.

Com relação às informações sobre os autores, $54 \%$ destes são do gênero masculino, esta característica se repete em todos os eventos considerados, exceto no Convibra em que o gênero feminino foi a maioria. Quanto à média de autores por artigo, destaca-se que a maioria da produção acadêmica analisada teve, no mínimo, 01 autor e 01 coautor e entre as IES em que os autores encontram-se vinculados, a Universidade Federal de Brasília apresenta o maior número de pesquisadores a ela ligados.

Levantaram-se também os principais autores responsáveis pela produção científica no período, onde identificou-se Alberto Shigueru Matsumoto como principal autor, seguido por Abdelkader Bourahli e Kelmara Mendes Vieira. Portanto, a coleta de dados realizada, evidenciou que os temas finanças pessoais e educação financeira ainda são abordados timidamente nos eventos nacionais na área da Administração no Brasil, sendo pesquisados por um grupo pequeno de autores.

Esta conclusão pode ser reforçada através da verificação da rede de autoria construída por meio dos trabalhos analisados. A referida rede apresentou três grandes redes de autoria, envolvendo 05 autores e 19 coautores, ou seja, 25\% do total de autores e $43 \%$ da produção acadêmica.

Como limitação do estudo menciona-se a dificuldade encontrada na busca de artigos que gerasse uma amostra satisfatória para fins de análise. Diante disso, a pesquisa foi direcionada a diversos eventos, alguns com uma produção superior a outros. Logo, esta disparidade limitou algumas análises por evento, sendo necessário trabalhar os dados no âmbito geral.

Ao findar esse trabalho, sugere-se que o mesmo seja replicado, no entanto, utilizando-se como base algum periódico ou indexador. Também é válido um estudo bibliométrico sobre os temas de forma individual. Além disso, uma pesquisa sobre o estado da arte também pode ser realizada, visto que contribuiria para a evolução das teorias aqui abordadas, visando a discussões com novas pesquisas em ambas as áreas. 


\section{REFERÊNCIAS}

APPOLINÁRIO, F. Dicionário de metodologia científica: um guia para a produção do conhecimento científico. 2. ed. São Paulo: Atlas, 2011.

AVDZEJUS, É. E.; SANTOS, A. C. dos; SANTANTA, J. O. de. Endividamento precoce: uma análise da concessão de crédito e dos fatores que influenciam no endividamento de jovens universitários da Faculdade UNIME no município de Lauro de Freitas/ BA. In: SIMPÓSIO DE EXCELÊNCIA EM GESTÃO E TECNOLOGIA, 9., Rio de Janeiro, 2012. Anais... Rio de Janeiro: SEGe'T, 2012.

BATISTA, A. C. A.; PIGATI, G. M. S.; SILVA, M. M. da. Finanças pessoais: análise de facilidade de crédito, educação financeira e planejamento financeiro dos acadêmicos de administração. In: CONGRESSO NACIONAL DE EXCELÊNCIA EM GESTÃO, 10., Rio de Janeiro, 2014. Anais... Rio de Janeiro: CNEG, 2014.

CONCEIÇÃO, K. C. da; BREYER, R.; PRETE, M. D. Finanças pessoais: estudo dos gastos e da propensão ao endividamento em estudantes do curso de administração da Unicastelo de Fernandópolis/SP. In: ENCONTRO DE PÓS-GRADUAÇÃO E INICIAÇÃO CIENTÍfICA, 1., São Paulo, 2013, Anais... São Paulo: Unicastelo, 2013.

ESPEJO, M. M. dos S. B.; CRUZ, A. P. C. da; LOURENÇO, R. L.; ANTONOVZ, T.; ALMEIDA, L. B. de. Estado da arte da pesquisa contábil: um estudo bibliométrico de periódicos nacional e internacionalmente veiculados entre 2003 e 2007 . Revista de Informação Contábil, Recife, v. 3, n. 3, p. 94-116, jul./set. 2009.

FERNANDES, B. V. R.; MONTEIRO, D. L.; SANTOS, W. R. dos. Finanças pessoais: um estudo dos seus princípios básicos com alunos da Universidade de Brasília. CAP Accounting and Management, Curitiba, v. 6, n. 6, p. 9-28, 2012.

FIGUEIRA, R. F. Devo, não nego, pago quando puder: uma análise acerca dos fatores condicionantes no comportamento do consumidor endividado. 2013. $141 \mathrm{f}$. Dissertação (Mestrado em Administração) - Programa de Pós-graduação em Administração -Universidade Federal da Paraíba, João Pessoa, 2013. 
FLORES, S. A. M.; CAMPARA, J. P.; VIEIRA, K. M. Propensão ao endividamento no município de Santa Maria (RS): análise da influência da educação financeira e de variáveis demográficas. In: SEMINÁRIOS EM ADMINISTRAÇÃO, 15., São Paulo, 2012. Anais... São Paulo: SEMEAD, 2012.

G20 - Grupo dos 20. G20 Leaders Declaration. 2012. Disponível em: < http:// www.consilium.europa.eu/uedocs/cms_data/docs/pressdata/en/ec/131069.pdf $>$. Acesso em: 05 maio 2015.

GIORDANI, R. J. Como administrar as finanças pessoais. Joinville: Alphagraphics, 2014.

GOMES, D. M.; SORATO, K. A. D. L. Planejamento e controle das finanças pessoais com enfoque na utilização das ferramentas e serviços contábeis: um estudo com profissionais autônomos. In: SEMINÁRIO DE CIÊNCIAS SOCIAIS APLICADAS, 2., Criciúma, 2010. Anais... Criciúma: UNESC, 2010.

GRIFONI, A.; MESSY, F. Current status of national strategies for financial education: a comparative analysis and relevant practices. OECD Working Papers on Finance, Insurance and Private Pensions, n. 16, p. 1-36, 2012.

GRÜSSNER, P. M. Administrando as finanças pessoais para criação de patrimônio. 2007. 102 f. Trabalho de Conclusão de Curso (Graduação em Administração) Departamento de Ciências Administrativas - Universidade Federal do Rio Grande do Sul, Porto Alegre, 2007.

GUIMARÃES, L. P. Educação financeira em três capítulos. São Paulo: PerSe, 2013.

INFE. International Gateway Financial Education. 2015. Disponível em: < http:// www.financial-education.org/join_INFE.html > . Acesso em: 05 maio 2015.

IPEA - Instituto de Pesquisa Econômica Aplicada. IEF: Índice de Expectativas das Famílias. 2012. Disponível em: < http://www.ipea.gov.br/agencia/images/stories/PDFs/ IEF/120717_ief_23.pdf>. Acesso em: 25 abr. 2015. 
ISMAIL, S.; KAMIS, R.; HASHIM, N.; HARUN, H.; KHAIRUDDIN, N. S. An empirical investigation on determinants of attitude towards saving behavior. Procedia Economics and Finance, International Conference on Economics and Business Research, v. 7, p. 1-242, 2013.

KAPOOR, J. R.; DALABAY, L. R.; HUGHES, R. J. Personal finance. 10. ed. New York: McGraw- Hill, 2012.

KIYOSAKI, R. O poder da educação financeira: lições sobre dinheiro que não se aprendem na escola. Rio de Janeiro: Elsevier, 2011.

KRUMMENAUER, L. D. Educação financeira para adolescentes do ensino médio em Sapucaia do Sul. 2011. 154 f. Trabalho de Conclusão de Curso (Graduação em Ciências Contábeis) - Unidade Acadêmica de Graduação - Universidade do Vale do Rio dos Sinos, São Leopoldo, 2011.

LUCENA, W. G. L.; MARINHO, R. A. de L. Competências financeiras: uma análise das decisões financeiras dos discentes no tocante as finanças pessoais. In: In: SEMINÁRIOS EM ADMINISTRAÇÃO, 16., São Paulo, 2013. Anais... São Paulo: SEMEAD, 2013.

LUCKE, V. A. C.; FILIPIN, R.; BRIZOLLA, M. M. B.; VIEIRA, E. P. Comportamento financeiro pessoal: um comparativo entre jovens e adultos de uma cidade da região noroeste do Estado do RS. In: SEMINÁRIOS EM ADMINISTRAÇÃO, 17., São Paulo, 2014. Anais... São Paulo: SEMEAD, 2014.

LUSARDI, A.; MITCHELL, O. S.; CURTO, V. Financial literacy among the young. Journal of Consumer Affairs, v. 44, n. 2, p. 358-380, 2010.

MARCOLIN, S.; ABRAHAM, A. Financial literacy research: current literature and future opportunities. In: INTERNATIONAL CONFERENCE OF CONTEMPORARY BUSINESS, 3., Australia, 2006. Annals... Australia: Faculty of Commerce, 2006.

MARQUES, E. V.; SOUZA, A. C. A. de; PESSOA, Y. B. Análise da gestão financeira pessoal de gestores e micro empreendedores do município de Fortaleza - Ceará a luz das finanças comportamentais. In: SIMPÓSIO DE ADMINISTRAÇÃO DA PRODUÇÃO, 
LOGÍSTICA E OPERAÇÕES INTERNACIONAIS, 17., São Paulo, 2014. Anais... São Paulo: SIMPOI, 2014.

MEDEIROS, F. S. B.; LOPES, T. de A. M. Finanças pessoais: um estudo com alunos do curso de ciências contábeis de uma IES privada de Santa Maria - RS. Revista Eletrônica de Estratégia \& Negócios, Florianópolis, v. 7, n. 2, p. 221-251, maio/ ago. 2014.

MELLO, W. Educação financeira. Joinville: Ebook, 2013.

METTE, F. M. B.; MATOS, C. A. de. Comportamento do consumidor endividado: um estudo com indivíduos de baixa renda. In: ENCONTRO DA ANPAD, 38., Rio de Janeiro, 2014. Anais... Rio de Janeiro: EnANPAD, 2014.

MICHEL, M. H. Metodologia e pesquisa científica em ciências sociais: um guia prático para acompanhamento da disciplina e elaboração de trabalhos monográficos. 2. ed. São Paulo: Atlas, 2009.

OECD - Organization for Economic Co-operation and Development. PISA 2012 assessment and analytical framework: mathematics, reading, science, problem solving and financial literacy. OECD Publishing, 2013.

OLIVEIRA, R. B. de; KASPCZAK, M. C. de M. Planejamento financeiro pessoal: uma revisão bibliográfica. In: CONGRESSO INTERNACIONAL DE ADMINISTRAÇÃO, Ponta Grossa, 2013. Anais... Ponta Grossa, 2013.

OPLETALOVÁ, A. Financial education and financial literacy in the Czech education system. Procedia - Social and Behavioral Sciences, v. 171, p. 1176-1184, 2015.

PINTO, N. G. M.; CORONEL, D. A. Abordagem do endividamento, superendividamento e inadimplência nas capitais brasileiras (2010-2012). In: CONGRESSO INTERNACIONAL DE ADMINISTRAÇÃO, Ponta Grossa, 2013. Anais... Ponta Grossa, 2013.

POTRICH, A. C. G.; VIEIRA, K. M.; KIRCH, G. Determinantes da alfabetização financeira: proposição de um modelo e análise da influência das variáveis socioeconômi- 
cas e demográficas. In: ENCONTRO DA ANPAD, 38., Rio de Janeiro, 2014. Anais... Rio de Janeiro: EnANPAD, 2014.

PUNHAGUI, B. C.; VIEIRA, S. F. A. Educação financeira e decisões de consumo: um estudo de caso no Instituto Agronômico do Paraná. In: SEMINÁRIOS EM ADMINISTRAÇÃO, 16., São Paulo, 2013. Anais... São Paulo: SEMEAD, 2013.

SAVOIA, J. R. F.; SAITO, A. T.; SANTANA, F. de A. Paradigmas da educação financeira no Brasil. Revista de Administração Pública, Rio de Janeiro, v. 41, n. 6, p. 11211141, nov./dez. 2007.

SOUSA, E. R. de; ARAUJO, F. de A. L.; FAGUNDES, J. V. de A.; ÂNGULO, M. J.; SOUZA, M. A. P. de; ORSI, R. V.; MATTA, R. O. B.; BISI, R. M. Caderno de educação financeira - gestão de finanças pessoais (conteúdo básico). Banco Central do Brasil: Brasília, 2013.

SOUZA, A. F. de.; TORRALVO, C. F. A gestão dos próprios recursos e a importância do planejamento financeiro pessoal. In: SEMINÁRIOS EM ADMINISTRAÇÃO, 7., São Paulo, 2004. Anais... São Paulo: SEMEAD, 2004.

SOUZA, R. C. de; SILVA, F. de S. B.; BARROS, I. M.; QUEIROZ, M. das G. M. A importância da educação financeira no contexto atual: a realidade dos bairros Riacho do Meio e Manoel Deodato em Pau dos Ferros-RN. Revista Extendere, Mossoró, v. 1, n. 1, p. 180-194, jan./jun. 2013.

WISNIEWSKI, M. L. G. A importância da educação financeira na gestão das finanças pessoais: uma ênfase na popularização do mercado de capitais brasileiro. Revista Intersaberes, Curitiba v. 6, n. 11, p. 155-172, 2011.

WOHLEMBERG, T. R.; BRAUM, L. M. dos S.; ROJO, C. A. Finanças pessoais: uma pesquisa com os acadêmicos da Unioeste Campus de Marechal Cândido Rondon. Ciências Sociais Aplicadas em Revista, Cascavel, v. 11, n. 21, p. 133-152, 2011. 